\title{
Modified generalised successive over-relaxation method for augmented linear systems
}

Sheng-Kun Li $^{1} \quad$ Ting-Zhu Huang ${ }^{2} \quad$ Li-Tao Zhang ${ }^{3}$

(Received 4 October 2008; revised 12 May 2010)

\begin{abstract}
We introduce here a modified generalised successive over-relaxation (MGSOR) method to solve augmented linear systems. We prove that the MGSOR method converges to the unique solution of the linear system for a loose restriction on three parameters. Finally, a numerical example illustrates the effectiveness of the MGSOR iteration method which outperforms the modified sor-like method and the generalised successive over-relaxation method.
\end{abstract}

\section{Contents}

1 Introduction

E98

2 The MGSOR method

E100

3 Convergence region of MGSOR method

E104

http://anziamj . austms.org.au/ojs/index.php/ANZIAMJ/article/view/1551 gives this article, (C) Austral. Mathematical Soc. 2010. Published May 24, 2010. ISSN 1446-8735. (Print two pages per sheet of paper.) 


\section{Introduction}

Let $A \in \mathbb{R}^{m \times m}$ be a symmetric positive definite matrix and $B \in \mathbb{R}^{m \times n}$ be a matrix of full column rank, when $m \geqslant n$. Denote by $B^{\top}$ the transpose of matrix B. Then the augmented linear system is of the form

$$
\left(\begin{array}{cc}
A & B \\
B^{T} & 0
\end{array}\right)\left(\begin{array}{l}
x \\
y
\end{array}\right)=\left(\begin{array}{l}
b \\
q
\end{array}\right)
$$

where $\mathbf{b} \in \mathbb{R}^{\mathrm{m}}$ and $\mathbf{q} \in \mathbb{R}^{\mathfrak{n}}$ are two given vectors.

The system of linear equations (1) corresponds to the Kuhn-Tucker conditions for linearly constrained quadratic programming problems or saddle point problems. Such systems typically result from mixed or hybrid finite element approximations of second order elliptic problems, elasticity problems or the Stokes equations [4] and from Lagrange multiplier methods [5].

Since the above problem is large and sparse, iterative methods for solving equation (1) are effective because of storage requirements and preservation of sparsity. Recently, Golub et al. [2] proposed a soR-like method to solve the system (1).

Method 1.1: The SOR-like method Let $\mathrm{Q} \in \mathbb{R}^{n \times n}$ be a nonsingular and symmetric matrix. Give initial vectors $x^{(0)} \in \mathbb{R}^{m}$ and $y^{(0)} \in \mathbb{R}^{n}$, and a relaxation factor $\omega>0$. For $k=0,1,2, \ldots$ until the iteration sequence $\left\{\left(x^{(k)^{\top}}, y^{(k)^{\top}}\right)^{\top}\right\}$ is convergent, compute

$$
\left\{\begin{array}{l}
x^{(k+1)}=(1-\omega) x^{(k)}+\omega A^{-1}\left(b-B y^{(k)}\right) \\
y^{(k+1)}=y^{(k)}+\omega Q^{-1}\left(B^{\top} x^{(k+1)}-q\right) .
\end{array}\right.
$$


Here, $\mathrm{Q}$ is an approximate (preconditioning) matrix of the Schur complement matrix $B^{\top} A^{-1} B$.

Golub et al. [2] proved the convergence of the sor-like method and determined its optimal iteration parameters as well as the corresponding optimal convergence factor.

To further improve the convergence speed of the sor-like method, Bai et al. [1] presented a generalised successive over-relaxation (GSOR) method for the augmented linear system (1).

Method 1.2: The GSOR method Let $Q \in \mathbb{R}^{n \times n}$ be a nonsingular and symmetric matrix. Give initial vectors $x^{(0)} \in \mathbb{R}^{m}$ and $y^{(0)} \in \mathbb{R}^{n}$, and two relaxation factors $\omega, \tau \neq 0$. For $k=0,1,2, \ldots$ until the iteration sequence $\left\{\left(x^{(k)^{\top}}, y^{(k)^{\top}}\right)^{\top}\right\}$ is convergent, compute

$$
\left\{\begin{array}{l}
x^{(k+1)}=(1-\omega) x^{(k)}+\omega A^{-1}\left(b-B y^{(k)}\right), \\
y^{(k+1)}=y^{(k)}+\tau Q^{-1}\left(B^{\top} x^{(k+1)}-q\right) .
\end{array}\right.
$$

Here, $\mathrm{Q}$ is an approximate (preconditioning) matrix of the Schur complement matrix $B^{\top} A^{-1} B$.

Bai et al. [1] also proved the convergence of the GSOR method, and determined its optimal iteration parameter as well as the corresponding optimal convergence factor.

The GSOR method is modified here by introducing an additional parameter. Section 2 establishes a modified GSOR (MGSOR for short) method for the augmented system (1). Section 3 discusses the convergence region for the method. Numerical results of Section 4 show that the MGSOR method is more effective than the MSOR-like method and the GSOR method for solving the augmented linear system. 


\section{The MGSOR method}

For the sake of simplicity, we rewrite the augmented system (1) as

$$
\left(\begin{array}{cc}
A & B \\
-B^{T} & 0
\end{array}\right)\left(\begin{array}{l}
x \\
y
\end{array}\right)=\left(\begin{array}{c}
b \\
-q
\end{array}\right)
$$

We make the following splitting

$$
\mathbb{A} \equiv\left(\begin{array}{cc}
A & B \\
-B^{\top} & 0
\end{array}\right)=D-L-U
$$

where

$$
\mathrm{D}=\left(\begin{array}{cc}
\mathrm{A} & 0 \\
0 & \mathrm{Q}
\end{array}\right), \quad \mathrm{L}=\left(\begin{array}{cc}
0 & 0 \\
\mathrm{~B}^{\top} & \alpha \mathrm{Q}
\end{array}\right), \quad \mathrm{U}=\left(\begin{array}{cc}
0 & -\mathrm{B} \\
0 & (1-\alpha) \mathrm{Q}
\end{array}\right)
$$

and $\mathrm{Q} \in \mathbb{R}^{\mathfrak{n} \times n}$ is a prescribed nonsingular and symmetric matrix. Let $\omega$ and $\tau$ be two nonzero reals, $I_{m} \in \mathbb{R}^{m \times m}$ and $I_{m} \in \mathbb{R}^{n \times n}$ be the $m \times m$ and the $\mathrm{n} \times \mathrm{n}$ identity matrices, respectively, and

$$
\Omega=\left(\begin{array}{cc}
\omega \mathrm{I}_{\mathrm{m}} & 0 \\
0 & \tau \mathrm{I}_{\mathrm{n}}
\end{array}\right)
$$

Then we consider the following MGSOR method with three parameters for solving the augmented linear system (1)

$$
(D-\Omega L)\left(\begin{array}{c}
x^{(k+1)} \\
y^{(k+1)}
\end{array}\right)=[(I-\Omega) D+\Omega U]\left(\begin{array}{c}
x^{(k)} \\
y^{(k)}
\end{array}\right)+\Omega\left(\begin{array}{c}
b \\
-q
\end{array}\right),
$$

where I is the identity matrix.

Evidently, when $\alpha=0$ the MGSOR method becomes the GSOR method [1], and when $\omega=\tau$ it becomes the MSOR-like method [3]. With a proper choice 
of the parameters, the MGSOR method will converge faster than the MSOR-like method and the GSOR method in certain cases. Note that

$$
(D-\Omega L)=\left(\begin{array}{cc}
A & 0 \\
-\tau B^{T} & (1-\tau \alpha) Q
\end{array}\right)
$$

Since matrix $A$ is symmetric positive definite and matrix $Q$ is nonsingular, therefore

$$
\operatorname{det}(D-\Omega L)=(1-\tau \alpha)^{n} \operatorname{det}(A) \operatorname{det}(Q) \neq 0
$$

if and only if

$$
1-\tau \alpha \neq 0 \quad \text { or } \quad \tau \alpha \neq 1 \text {. }
$$

From now on we assume that the parameters $\tau$ and $\alpha$ satisfy equation (3). The iterative matrix $\mathrm{H}_{(\omega, \tau, \alpha)}$ of the MGSOR method is shown as

$$
\begin{aligned}
\mathrm{H}_{(\omega, \tau, \alpha)} & \equiv(D-\Omega L)^{-1}[(I-\Omega) D+\Omega U] \\
& =\left(\begin{array}{cc}
(1-\omega) I_{m} & -\omega A^{-1} B \\
\frac{(1-\omega) \tau}{1-\tau \alpha} Q^{-1} B^{\top} & I_{n}-\frac{\omega \tau}{1-\tau \alpha} Q^{-1} B^{\top} A^{-1} B
\end{array}\right) .
\end{aligned}
$$

Let $\lambda$ be an eigenvalue of $\mathrm{H}_{(\boldsymbol{\omega}, \tau, \alpha)}$ and $\left(\boldsymbol{u}^{\top}, \nu^{\top}\right)^{\top}$ be the corresponding eigenvector, we have

$$
\mathrm{H}_{(\omega, \tau, \alpha)}\left(\begin{array}{l}
u \\
v
\end{array}\right)=\lambda\left(\begin{array}{l}
u \\
v
\end{array}\right)
$$

or

$$
[(I-\Omega) D+\Omega U]\left(\begin{array}{l}
u \\
v
\end{array}\right)=\lambda(D-\Omega L)\left(\begin{array}{l}
u \\
v
\end{array}\right) .
$$

Equation (5) is equivalent to

$$
(1-\omega-\lambda) u=\omega A^{-1} B v \text { and }(\lambda-1)(1-\tau \alpha) v=\lambda \tau Q^{-1} B^{\top} u .
$$

Lemma 1 If $\lambda$ is an eigenvalue of $\mathrm{H}_{(\omega, \tau, \alpha)}$, then $\lambda \neq 1$. 
Proof: If $\lambda=1$ and the associated eigenvector is $\left(u^{\top}, v^{\top}\right)^{\top}$, then it follows from system (6) and $\omega \neq 0$ that

$$
-u=A^{-1} B v \text { and } Q^{-1} B^{\top} u=0,
$$

resulting in $Q^{-1} B^{\top} A^{-1} B v=0$. Since $Q^{-1} B^{\top} A^{-1} B$ is nonsingular, then we have $v=0$ and $u=0$. This contradicts the condition that $\left(u^{\top}, v^{\top}\right)^{\top}$ is an eigenvector of the iteration matrix $\mathrm{H}_{(\omega, \tau, \alpha)}$. Hence $\lambda \neq 1$, concluding the proof.

Lemma 2 If $\mathrm{m}>\mathrm{n}$, then $\lambda=1-\omega$ is an eigenvalue of $\mathrm{H}_{(\omega, \tau, \alpha)}$ with a multiplicity of at least $\mathrm{m}-\mathrm{n}$; if $\mathrm{m}=\mathrm{n}$, then $\lambda=1-\omega$ is not an eigenvalue of $\mathrm{H}_{(\omega, \tau, \alpha)}$ when $\omega \neq 1$, and is an eigenvalue of $\mathrm{H}_{(\omega, \tau, \alpha)}$ with a multiplicity of at least $\mathrm{m}$ when $\omega=1$.

Proof: First, we assume $\omega \neq 1$. If $\lambda=1-\omega$ is an eigenvalue of $H_{(\omega, \tau, \alpha)}$, then there exists a non-zero vector $\left(u^{\top}, v^{\top}\right)^{\top}$ which satisfies equation (5) with $\lambda=1-\omega$. Therefore, by system (6) and $\omega \neq 0$ we have

$$
A^{-1} B v=0, \quad \text { and } \quad-\omega(1-\tau \alpha) v=(1-\omega) \tau Q^{-1} B^{\top} u .
$$

Since matrix $B$ has a full column rank and $\tau \neq 0$, the above system is equivalent to

$$
v=0 \text { and } \mathrm{Q}^{-1} \mathrm{~B}^{\mathrm{T}} \mathrm{u}=0 .
$$

$B^{\top}$ is an $n \times m$ matrix of $\operatorname{rank}\left(B^{\top}\right)=n$. Therefore if $m>n, Q^{-1} B^{\top} u=0$ has $m-n(>0)$ independent non-zero solutions, whereas if $m=n, Q^{-1} B^{\top} u=0$ has no non-zero solutions. Thus $\lambda=1-\omega$ is not only an eigenvalue of $H_{(\omega, \tau, \alpha)}$ but is also an eigenvalue with multiplicity of $m-n$ if $m>n$. However, if $m=n, \lambda=1-\omega$ is not an eigenvalue of $H_{(\omega, \tau, \alpha)}$. Thus the lemma is true when $\omega \neq 1$.

Next we assume that $\omega=1$ and we have

$$
\mathrm{H}_{(\omega, \tau, \alpha)}=\mathrm{H}_{(1, \tau, \alpha)}=\left(\begin{array}{cc}
0 & -\omega A^{-1} B \\
0 & \mathrm{I}_{n}-\frac{\omega \tau}{1-\tau \alpha} \mathrm{Q}^{-1} \mathrm{~B}^{\top} \mathrm{A}^{-1} \mathrm{~B}
\end{array}\right) .
$$


Note that the zero matrix ( 0 ) in the main diagonal position is $\mathfrak{m} \times \mathfrak{m}$. Hence $\lambda=1-\omega=0$ is an eigenvalue of the iteration matrix $\mathrm{H}_{(\omega, \tau, \alpha)}$ with at least multiplicity of $m$, which shows the lemma holds for $\omega=1$. Thus Lemma 2 is proved.

Note from system (6) that if $\lambda$ and $\left(\mathbf{u}^{\top}, v^{\top}\right)^{\top}$ are the eigenvalue and eigenvector of $\mathrm{H}_{(\omega, \tau, \alpha)}$, and if $\lambda \neq 1-\omega$, then $v \neq 0$. Now we prove the following theorem.

Theorem 3 Let $\mathrm{H}_{(\omega, \tau, \alpha)}$ be the iteration matrix of the MGSOR method. Then (i) $\lambda=1-\omega$ is an eigenvalue of $\mathrm{H}_{(\omega, \tau, \alpha)}$ if $\mathrm{m}>\mathrm{n}$. (ii) For any eigenvalue $\lambda \neq 1-\omega$ of $\mathrm{H}_{(\omega, \tau, \alpha)}$, there is an eigenvalue $\mu$ of $\mathrm{Q}^{-1} \mathrm{~B}^{\top} \mathrm{A}^{-1} \mathrm{~B}$ such that $\lambda$, $\mu, \omega, \tau$ and $\alpha$ satisfy the functional equation

$$
(1-\omega-\lambda)(\lambda-1)(1-\tau \alpha)=\lambda \omega \tau \mu .
$$

(iii) For any eigenvalue $\mu$ of $\mathrm{Q}^{-1} \mathrm{~B}^{\top} \mathrm{A}^{-1} \mathrm{~B}$, if $\lambda \neq 1-\omega$ and $\lambda, \mu, \omega$, $\tau$ and $\alpha$ satisfy the above functional equation, then $\lambda$ is an eigenvalue of $\mathrm{H}_{(\omega, \tau, \alpha)}$.

Proof: Conclusion (i) comes from Lemma 2.

For conclusion (ii), we let $\lambda \neq 1-\omega$ be the eigenvalue of the $\mathrm{H}_{(\omega, \tau, \alpha)}$ and $\left(u^{\top}, v^{\top}\right)^{T}$ be the corresponding eigenvector. Then they satisfy system (6). Thus we have

$$
(1-\omega-\lambda)(\lambda-1)(1-\tau \alpha) v=\lambda \omega \tau \mathrm{Q}^{-1} \mathrm{~B}^{\top} \mathrm{A}^{-1} \mathrm{~B} v .
$$

Since $\lambda \neq 1-\omega, \lambda \neq 1$ by Lemma 1 , and $\nu \neq 0$ by the note after Lemma 2 , $1-\tau \alpha \neq 0$ (equation (3)). Therefore $\lambda \omega \tau \neq 0$. Thus there is an eigenvalue, say $\mu$, of $Q^{-1} B^{\top} A^{-1} B$ such that $\lambda, \mu, \omega, \tau$ and $\alpha$ satisfy equation (8), concluding the proof of conclusion (ii).

For conclusion (iii), we let $\mu$ and $v(\neq 0)$ be the eigenvalue and eigenvector of the matrix $Q^{-1} B^{\top} A^{-1} B$. Therefore we have $Q^{-1} B^{\top} A^{-1} B v=\mu \nu$. By the 
conditions of the theorem, equation (9) holds. If we let

$$
u=\frac{\omega}{1-\omega-\lambda} A^{-1} B v,
$$

system (6) holds, which is equivalent to system (8). Thus $\lambda$ is an eigenvalue of $\mathrm{H}_{(\omega, \tau, \alpha)}$, since $\left(\mathbf{u}^{\top}, v^{\top}\right)^{\top}$ is non-zero, resulting in the last part of the theorem. Thus the theorem is proved.

From Theorem 3 we have the following corollary.

Corollary 4 Let $\rho\left(\mathrm{H}_{(\omega, \tau, \alpha)}\right)$ be the spectral radius of the MGSOR iteration matrix $\mathrm{H}_{(\omega, \tau, \alpha)}$ and $\mathrm{m}>\mathrm{n}$, then

$$
\rho\left(H_{(\omega, \tau, \alpha)}\right) \geqslant|1-\omega| .
$$

By Corollary 1, the necessary condition for the convergence of the MGSOR method when $m>n$ is

$$
0<\omega<2 \text {. }
$$

\section{Convergence region of MGSOR method}

Next we study the convergence region for parameters $\omega, \tau$ and $\alpha$ in the MGSOR method for solving the augmented system (1). For this, we first quote the following lemma which will be used later.

Lemma 5 (Young [7]) Consider the quadratic equation $\mathrm{x}^{2}-\mathrm{bx}+\mathrm{c}=0$, when $\mathrm{b}$ and $\mathrm{c}$ are real numbers. Both roots of the equation are less than one in modulus if and only if $|\mathrm{c}|<1$ and $|\mathrm{b}|<1+\mathrm{c}$.

Theorem 6 We assume that the parameters $\tau$ and $\alpha$ satisfy equation (3). Let $\mathrm{A} \in \mathbb{R}^{\mathrm{m} \times \mathrm{m}}$ and $\mathrm{Q} \in \mathbb{R}^{\mathrm{n} \times \mathrm{n}}$ be symmetric positive definite, and $\mathrm{B} \in \mathbb{R}^{\mathrm{m} \times \mathrm{n}}$ be of full column rank. Denote the smallest and the largest eigenvalues of the 
matrix $\mathrm{Q}^{-1} \mathrm{~B}^{\top} \mathrm{A}^{-1} \mathrm{~B}$ by $\mu_{\min }$ and $\mu_{\max }$, respectively. Then the MGSOR method is convergent if $\omega$ satisfies $0<\omega<2$ and if $\tau$ and $\alpha$ satisfy

$$
0<\frac{\tau}{1-\tau \alpha}<\frac{2(2-\omega)}{\omega \mu_{\max }}
$$

Proof: Evidently, we see that all eigenvalues $\mu$ of the matrix $Q^{-1} B^{\top} A^{-1} B$ are real and positive.

From Theorem 3, we have

$$
\lambda^{2}+\left(\omega-2+\frac{\omega \tau \mu}{1-\tau \alpha}\right) \lambda+(1-\omega)=0 .
$$

By Lemma $5,|\lambda|<1$ if and only if

$$
\left\{\begin{array}{l}
|1-\omega|<1 \\
\left|\omega-2+\frac{\omega \tau \mu}{1-\tau \alpha}\right|<2-\omega
\end{array}\right.
$$

which is equivalent to

$$
\left\{\begin{array}{l}
0<\omega<2 \\
0<\frac{\omega \tau \mu}{1-\tau \alpha}<2(2-\omega)
\end{array}\right.
$$

Then

$$
\left\{\begin{array}{l}
0<\omega<2 \\
0<\frac{\tau}{1-\tau \alpha}<\frac{2(2-\omega)}{\omega \mu_{\max }} .
\end{array}\right.
$$

This completes the proof of Theorem 6 .

Remark When $\alpha=0$ this theorem becomes Theorem 2.1 by Bai et al. [1]. The convergence domain for the parameter $\tau$ of the MGSOR method is greater than the convergence domain of the GSOR method when $\alpha$ is properly chosen. When $\omega=\tau$, this theorem becomes Theorem 2 by Shao [3]. 


\section{TABLE 1: Choices of matrix Q.}

\begin{tabular}{cll}
\hline Case no. & Matrix Q & Description \\
\hline 1 & $\mathrm{~B}^{\top} \hat{\mathrm{A}}^{-1} \mathrm{~B}$ & $\hat{\mathrm{A}}=\operatorname{diag}(\mathrm{A})$ \\
2 & $\mathrm{~B}^{\top} \hat{\mathrm{A}}^{-1} \mathrm{~B}$ & $\hat{\mathrm{A}}=\operatorname{tridiag}(\mathrm{A})$ \\
3 & $\operatorname{tridiag}\left(\mathrm{B}^{\top} \hat{\mathrm{A}}^{-1} \mathrm{~B}\right)$ & $\hat{\mathrm{A}}=\operatorname{tridiag}(\mathrm{A})$ \\
\hline
\end{tabular}

\section{Numerical example}

The MGSOR method, the GSOR method and the MSOR-like method were used to solve the following augmented linear system, respectively.

Example (Darvishi and Hessari [6]). Consider the augmented linear system (1), in which

$$
\begin{aligned}
& A=\left(\begin{array}{cc}
I \otimes T+T \otimes I & 0 \\
0 & I \otimes T+T \otimes I
\end{array}\right) \in \mathbb{R}^{2 p^{2} \times 2 p^{2}}, \\
& B=\left(\begin{array}{c}
I \otimes F \\
F \otimes I
\end{array}\right) \in \mathbb{R}^{2 p^{2} \times p^{2}} \\
& T=\frac{1}{h^{2}} \cdot \operatorname{tridiag}(-1,2,-1) \in \mathbb{R}^{p \times p}, \\
& F=\frac{1}{h} \cdot \operatorname{tridiag}(-1,1,0) \in \mathbb{R}^{p \times p},
\end{aligned}
$$

with $\otimes$ being the Kronecker product symbol, $h=1 /(p+1)$ the discretization meshsize, and $S=\operatorname{tridiag}(a, b, c)$ is tridiagonal matrix with $S_{i, i}=b, S_{i+1, i}=$ $a$ and $S_{i, i+1}=c$ for appropriate $i$.

For this example, we set $m=2 p^{2}$ and $n=p^{2}$. Hence, the total number of variable is $m+n=3 p^{2}$. We chose the matrix $Q$ as an approximation to the matrix $B^{\top} A^{-1} B$, according to the cases listed in Table 1 .

In our computations, all runs with respect to the MSOR-like method, the GSOR method and the MGSOR method were started from the initial vec- 
tor $\left(x^{(0)^{\top}}, y^{(0)^{\top}}\right)^{\mathrm{T}}=0$, and terminated when the current iteration satisfied $\left\|r_{k}\right\|<10^{-6}$, where $r_{k}$ is the $k$ th residual of the iterations. We chose the right hand side vector $\left(b^{\top}, q^{\top}\right)^{\top} \in \mathbb{R}^{m+n}$ such that the exact solution of the augmented system $(1)$ is $\left(\left(x^{*}\right)^{\top},\left(y^{*}\right)^{\top}\right)^{\top}=(1,1, \ldots, 1) \in \mathbb{R}^{m+n}$. The optimum parameters for the GSOR method were determined according to the results of Bai et al. [1]. We chose the parameters for the Msor-like method and the MGSOR method by trial and error. We report the results in Table 2, for different values of $m$ and $n$, where IT denotes the number of iterations and $\rho$ represents the spectral radius.

All results show our method is better than the results obtained by the MSORlike method and the GSOR method with their corresponding optimal parameters. The parameters of the MGSOR method are not optimal and only lie in the convergence region of the method. The determination of the optimum parameters for the MGSOR method needs further study.

Acknowledgements The authors express their great gratitude to the referees and editor Dr B. D. Craven for their detailed and helpful suggestions for revising this paper. Supported by 973 Program (2007CB311002), NSFC (10926190, 60973015), Sichuan Province Sci. \& Tech. Project (2009GZ0004, 2009HH0025) and Sci. Found. of CUIT (CRF200806).

\section{References}

[1] Z. Z. Bai, B. N. Parlett, Z. Q. Wang, On generalized successive overrelaxation methods for augmented linear systems, Numer. Math., 102(2005), 1-38, doi:10.1007/s00211-005-0643-0 E99, E100, E105, E107

[2] G. H. Golub, X. Wu, J. Y. Yuan, SOR-like methods for augmented systems, BIT, 41(2001), 71-85, doi:10.1023/A:1021965717530 E98, E99 
TABLE 2: Iterations (IT) and $\rho$ for the Example.

\begin{tabular}{|c|c|c|c|c|c|}
\hline$m$ & & & 128 & 512 & 1152 \\
\hline$n$ & & & 64 & 256 & 576 \\
\hline$m+n$ & & & 192 & 768 & 1728 \\
\hline \multirow[t]{13}{*}{ Case 1} & \multirow[t]{4}{*}{ MSOR-like } & $\omega$ & 0.44 & 0.265 & 0.188 \\
\hline & & $\alpha$ & 0.2 & 0.2 & 0.2 \\
\hline & & $\rho$ & 0.7483 & 0.8573 & 0.9011 \\
\hline & & IT & 73 & 147 & 256 \\
\hline & \multirow[t]{4}{*}{ GSOR } & $\omega_{\text {opt }}$ & 0.5436 & 0.3419 & 0.2489 \\
\hline & & $\tau_{\mathrm{opt}}$ & 0.3751 & 0.2066 & 0.1423 \\
\hline & & $\rho_{\mathrm{opt}}$ & 0.6756 & 0.8112 & 0.8667 \\
\hline & & IT & 64 & 130 & 198 \\
\hline & \multirow[t]{5}{*}{ MGSOR } & $\omega$ & 0.54 & 0.341 & 0.244 \\
\hline & & $\tau$ & 0.351 & 0.198 & 0.14 \\
\hline & & $\alpha$ & 0.2 & 0.2 & 0.25 \\
\hline & & $\rho$ & 0.6782 & 0.8118 & 0.8695 \\
\hline & & IT & 53 & 105 & 163 \\
\hline \multirow[t]{13}{*}{ Case 2} & \multirow[t]{4}{*}{ MSOR-like } & $\omega$ & 0.5682 & 0.3539 & 0.255 \\
\hline & & $\alpha$ & 0.1 & 0.15 & 0.1 \\
\hline & & $\rho$ & 0.6571 & 0.8038 & 0.8631 \\
\hline & & IT & 50 & 101 & 157 \\
\hline & \multirow[t]{4}{*}{ GSOR } & $\omega_{\text {opt }}$ & 0.6633 & 0.4429 & 0.3307 \\
\hline & & $\tau_{\mathrm{opt}}$ & 0.4994 & 0.2854 & 0.1985 \\
\hline & & $\rho_{\mathrm{opt}}$ & 0.5803 & 0.7464 & 0.8181 \\
\hline & & IT & 45 & 91 & 138 \\
\hline & \multirow[t]{5}{*}{ MGSOR } & $\omega$ & 0.66 & 0.43 & 0.3285 \\
\hline & & $\tau$ & 0.455 & 0.27 & 0.19 \\
\hline & & $\alpha$ & 0.2 & 0.2 & 0.25 \\
\hline & & $\rho$ & 0.5831 & 0.7550 & 0.8195 \\
\hline & & IT & 38 & 78 & 114 \\
\hline \multirow[t]{13}{*}{ Case 3} & \multirow[t]{4}{*}{ MSOR-like } & $\omega$ & 0.94 & 0.95 & 0.95 \\
\hline & & $\alpha$ & 0.3 & 0.25 & 0.25 \\
\hline & & $\rho$ & 0.7671 & 0.8787 & 0.9151 \\
\hline & & IT & 62 & 128 & 188 \\
\hline & \multirow[t]{4}{*}{ GSOR } & $\omega_{\mathrm{opt}}$ & 0.7578 & 0.6314 & 0.5585 \\
\hline & & $\tau_{\mathrm{opt}}$ & 1.9508 & 2.5299 & 2.9743 \\
\hline & & $\rho_{\text {opt }}$ & 0.4922 & 0.6071 & 0.6644 \\
\hline & & IT & 35 & 53 & 68 \\
\hline & \multirow[t]{5}{*}{ MGSOR } & $\omega$ & 0.75 & 0.63 & 0.55 \\
\hline & & $\tau$ & 1.4 & 1.68 & 1.85 \\
\hline & & $\alpha$ & 0.2 & 0.2 & 0.2 \\
\hline & & $\rho$ & 0.5000 & 0.6083 & 0.6708 \\
\hline & & IT & 31 & 49 & 62 \\
\hline
\end{tabular}


[3] X. H. Shao, Z. Li, C. J. Li, Modified SOR-like method for the augmented system, Int. J. Comput. Math., 84(2007), 1653-1662, doi:10.1080/00207160601117313 E100, E105

[4] F. Brezzi, M. Fortin, Mixed and Hybrid Finite Element Methods, Springer-Verlag, New York and London, 1991. E98

[5] M. Fortin, R. Glowinski, Augmented Lagrangian Methods, Applications to the Numerical Solution of Boundary Value Problems, North-Holland, Amsterdam, 1983. E98

[6] M. T. Darvishi, P. Hessari, Symmetric SOR method for augmented systems, Appl. Math. Comput., 183(2006), 409-415, doi:10.1016/j.amc.2006.05.094 E106

[7] D. M. Young, Iterative Solution for Large Linear Systems, Academic Press, New York, 1971. E104

\section{Author addresses}

1. Sheng-Kun Li, School of Mathematical Sciences, University of Electronic Science and Technology of China, Chengdu 611731, P. R. China, and College of Math., Chengdu Univ. of Information Tech., Chengdu 610225, P. R. China. mailto:lishengkun@cuit.edu.cn

2. Ting-Zhu Huang, School of Mathematical Sciences, University of Electronic Science and Technology of China, Chengdu 611731, P. R. China.

mailto:tingzhuhuang@126.com

3. Li-Tao Zhang, Dept. of Math. Phy., Zhengzhou Institute of Aeronautical Industry Management, Zhengzhou, Henan 450015, P. R. China. 